\title{
Amortiguamiento en Estructuras de Acero Mediante Tratamiento a Cortante
}

\author{
Jhon Jairo Gil Peláez \\ Ph.D. in Civil Engineering, \\ University of Puerto Rico, Mayagüez Campus. \\ Docente Tiempo Completo, Investigador Grupo de Investigación \\ en Aplicaciones Mecatrónicas, GRAM, Universidad Santo Tomás \\ Bucaramanga, Colombia \\ decmeca@mail.ustabuca.edu.co
}

\author{
Luis E. Suárez \\ Ph.D. in Engineering Mechanics, \\ Engineering Science and Mechanics Department, \\ Virginia Polytechnic Institute \& State University \\ Professor and Coordinator of Structural Engineering \\ Department of Civil Engineering \\ University of Puerto Rico at Mayagüez, Puerto Rico \\ luis.suarez3@upr.edu
}

\begin{abstract}
Resumen- En este trabajo se evaluó la efectividad de los materiales viscoelásticos para disipar energía en edificios de acero por medio de simulaciones numéricas con elementos finitos usando el programa ABAQUS. El material viscoelástico se aplicó entre dos capas elásticas para crear un tratamiento a cortante. Las curvas de variación del factor de pérdida con respecto al parámetro de cortante para los tres primeros modos de vibración son construidas para la condición de apoyo impuesta por un edificio de corte. Se estudian diferentes combinaciones de espesores y se demuestra que aumentar el espesor de la capa del viscoelástica no implica necesariamente un aumento en la disipación de energía. Los resultados encontrados mostraron que el tratamiento a cortante no es efectivo cuando se aplica en las columnas de edificios de acero.
\end{abstract}

Palabras clave — Tratamiento a cortante, materiales viscoelásticos, disipación de energía, vigas emparedado.

\begin{abstract}
This work assesed the efectiveness of viscoelastic materials to dissipate energy in steel buildings, by means of numerical simulations with finite elements using program ABAQUS. A viscoleastic material layer was placed between two elastic layers to create a shear treatment. Loss factor variation curves versus shear parameter for the first three vibration modes are plotted for support conditions in a shear building. Different thickness combinations are studied, and it is shown that to increase the thickness of the viscoelastic layer not implies necessarily an increase in the energy dissipation. Results showed that shear treatment was not effective when applied to steel columns.
\end{abstract}

Keywords-Shear treatment, viscoelastic materials, energy dissipation, sandwich beams

\section{INTRODUCCIÓN}

Un material viscoelástico es un material que presenta tanto propiedades viscosas como elásticas. Estos materiales cuando son sometidos a una deformación, una parte de este trabajo se almacena y puede ser recuperado como energía elástica de deformación mientras que la otra parte se disipa en forma de calor sin deformaciones inelásticas (Christensen 1971). Los materiales amortiguadores se han usado mucho en ingeniería mecánica, aeronáutica y aeroespacial como un método para reducir las vibraciones transmitidas desde el motor, la turbina, la turbulencia y otros. Una medida del amortiguamiento que es capaz de proveer un material se puede obtener al considerar su capacidad de disipar energía bajo cargas cíclicas. La razón entre la energía disipada a la energía almacenada en un ciclo multiplicada por $1 / 2 p$ se conoce como factor de pérdida $\mathrm{h}$ y es un índice de la efectividad de un tratamiento de amortiguamiento.

En cuanto a ingeniería civil una posible aplicación sería la reducción de los efectos de los terremotos o del viento sobre las estructuras tanto de edificios como de puentes mediante un tratamiento de amortiguamiento superficial. Existen dos tipos de tratamiento básicos: el tratamiento de amortiguamiento "de capa libre" o "no restringida" o "extensional" en el cual la disipación de energía surge de la deformación extensional que se presenta en la viga cuando es sometida a una carga cíclica. La Fig. 1 muestra un esquema simple de este tratamiento. El otro tipo de tratamiento de amortiguamiento se conoce como "a cortante" o "de capa restringida". Éste consiste en usar una capa delgada de metal sobre el material viscoelástico. De este modo, en un tratamiento de amortiguamiento a cortante se forma un emparedado constituido por dos capas elásticas externas y una capa viscoelástica entre ellas, como se muestra en la Fig. 2. Cuando las dos capas externas ex- 
perimentan flexión cíclica deforman por cortante la capa viscoelástica. Ross et al. (1959) demostraron que el tratamiento de amortiguamiento a cortante es más eficiente que el tratamiento de amortiguamiento de capa libre. La mayor ventaja de los tratamientos de amortiguamiento es la reducción en la respuesta dinámica que se logra sin alterar significativamente la masa y rigidez de la estructura.

FIG. 1 VIGA CON TRATAMIENTO DE CAPA LIBRE
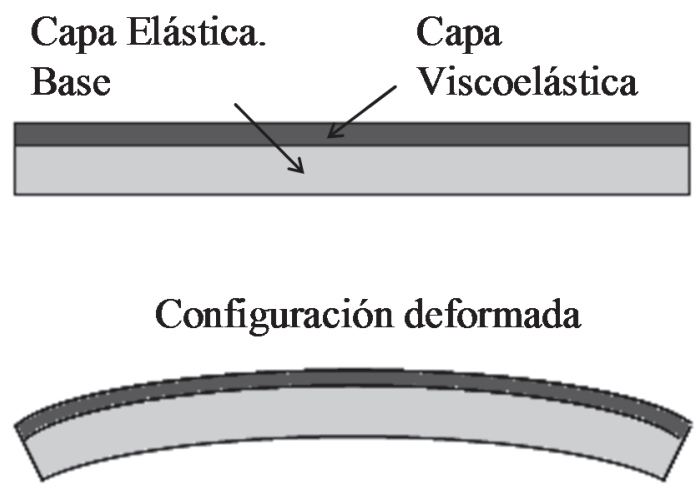

FIG. 2 VIGA LAMINADA DE TRES CAPAS. TRATAMIENTO DE CAPA RESTRINGIDA

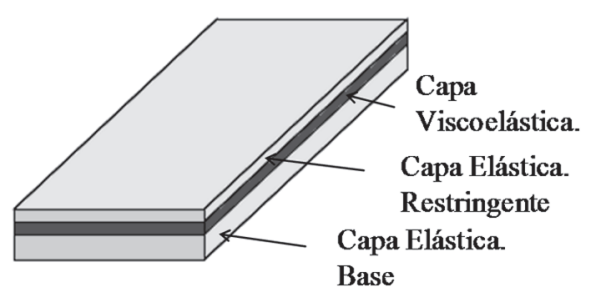

Configuración deformada

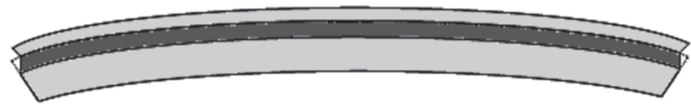

Una técnica que se puede usar para estudiar estructuras más complicadas con tratamiento de amortiguamiento es la modelación mediante elementos finitos. El método de elementos finitos ha sido usado por autores como Johnson y Kienholz (1982), Soni y Bogner (1982), Luengo (1991), Alberts et al. (1992) y Sun et al. (1990) para estudiar la respuesta de estructuras amortiguadas con geometrías tanto simples como complejas. Muchas de las investigaciones se limitaron a estudiar la respuesta de vigas de secciones rectangulares de un tramo y con condiciones de borde sencillos. No se ha encontrado en la literatura técnica buscada casos de modelos de una estructura en acero con secciones típicas como los perfiles W. Por lo tanto, en este trabajo se propone modelar con elementos finitos a través del programa ABAQUS una estructura de acero con tratamiento de capa restringida a lo largo de las columnas. El objetivo principal de esta investigación es estudiar la factibilidad de disminuir la respuesta a cargas dinámicas como vientos y terremotos. Para evaluar la efectividad de la técnica propuesta se calculan y examinan los factores de pérdida globales (o equivalentemente las razones de amortiguamiento) que se obtienen al colocar materiales amortiguadores en la estructura.

El tratamiento consistente en una capa de material viscoelástico adherida a la superficie de una estructura sometida a una carga cíclica fue estudiado primero por Oberts (1952) en una viga con una capa viscoelástica en una y en dos caras. La eficiencia del tratamiento no es alta, debido a que el material viscoelástico está sometido a deformaciones por tensión/compresión a lo largo del eje de la viga, y las deformaciones por cortante son muy bajas. Esta configuración también ha sido estudiada por otros autores como: Lifshitz y Leibowitz (1987), Plunkett y Lee (1969), Lunden (1980), Yildiz y Stevens (1985), Lumsdaine y Scott (1995) y Roy y Ganesan (1996).

El trabajo fundamental en el campo de tratamientos de amortiguamiento fue realizado por Ross et al. (1959) quienes desarrollaron la primera teoría para vigas tipo emparedado amortiguadas de tres capas y con material viscoelástico en el núcleo. La ecuación del movimiento para una viga tres capas ha sido obtenida por otros autores como: DiTaranto (1965), Mead y Markus (1969) y Rao (1978). Soni y Bogner (1982) estudiaron el tratamiento por cortante y desarrollaron un programa computacional con elementos finitos para analizar estructuras de naturaleza tridimensional y cáscaras. La efectividad del programa se verificó con tres ejemplos de interés práctico: una viga compuesta en voladizo, un tubo de escape de un motor jet y un panel cilíndrico. Johnson y Kienholz (1982) usaron el método de la energía modal implementado en NASTRAN aplicándolo a un número de elementos estructurales simples de tres capas, vigas, aros y placas. Los resultados obtenidos para las vigas se compararon con la teoría de cuarto orden de Ross et al. (1959) y con la sexto orden de DiTaranto (1965). Para las tres estructuras modeladas se encontró una buena coinciden- 
cia en los valores de las frecuencias naturales y los factores de pérdida modal con las soluciones disponibles. Algunos autores han estudiado los efectos de los cambios en la longitud, espesor, localización y factor de amortiguamiento de la capa viscoelástica, con el fin de lograr una configuración óptima que reduzca la respuesta dinámica de la viga. Sun et al. (1990) desarrollaron un modelo de elementos finitos para análisis dinámico de una viga en voladizo laminada de tres capas con núcleo de material viscoelástico. Los resultados obtenidos fueron comparados con experimentos realizados y mostraron que el modelo de elementos finitos desarrollado logra una representación eficiente y precisa del comportamiento de la viga. Concluyeron que el uso de tratamientos de amortiguamiento con capa de material viscoelástico reduce significativamente la respuesta dinámica de la estructura. También mostraron, que para cada modo y espesor de capa restringente, existe una longitud, localización y espesor de la capa viscoelástica que maximizan el amortiguamiento. Lifshitz y Leibowitz (1987) se propusieron desarroIlar una herramienta automática para el diseñador que le permitiera seleccionar las propiedades del material y las dimensiones de la viga, de tal manera que el diseño tenga el máximo amortiguamiento. El problema fue resuelto numéricamente para el factor de amortiguamiento modal y es un procedimiento iterativo que encuentra el máximo amortiguamiento para las condiciones de frontera y restricciones particulares como el peso, rigidez, espesor total y propiedades de los materiales, entre otros. Marceling et al. (1992) estudiaron el amortiguamiento óptimo de vigas con tratamiento de cortante cuando una o varias partes de la viga se cubren de material viscoelástico. Los autores demostraron que la máxima energía de deformación puede tomarse directamente como el principal objetivo del problema de optimización. Gil y Suárez (2007) estudiaron la formulación desarrollada por Rao y mediante simulaciones numéricas con elementos finitos, las combinaciones geométricas de una viga y el tratamiento a cortante con el fin de optimizar la disipación de energía. Mostraron que la disipación de energía que se logra al aplicar un tratamiento amortiguador de capa restringida a una viga está fuertemente asociada a las características geométricas de cada una de las partes que forman la viga emparedad.

\section{MODELAMIENTO DEL COMPORTAMIENTO VISCOELÁSTICO}

Los materiales viscoelásticos tienen la capacidad de liberar energía vía relajación y recuperación de las cadenas moleculares sometidas a deformación. La teoría clásica de elasticidad lineal para pequeñas deformaciones expresa los esfuerzos como proporcionales a la deformación; sin embargo, el comportamiento de un material viscoelástico también depende de la rapidez de cómo tiene lugar la deformación. Por lo tanto, este material no puede representarse mediante la ley de Hooke y se debe definir una relación más compleja entre los esfuerzos y deformaciones. Como su nombre lo indica, viscoelasticidad es una combinación de viscosidad y elasticidad. Si se restringe el estudio a materiales con comportamiento lineal, la componente viscosa puede ser modelada por un amortiguador con $\sigma=F \cdot \dot{\varepsilon}$, con $\dot{\varepsilon}=d \varepsilon / d t$ y la componente elástica con un resorte lineal con $\sigma=$ E $\varepsilon$ (Flugge 1967). Las cantidades E y F son parámetros del material. De acuerdo con las combinaciones de los elementos básicos se pueden obtener varios modelos: Modelo de Maxwell, Modelo de Kelvin-Voigt, Modelo Lineal Estándar y el Modelo Generalizado. El comportamiento viscoelástico de los materiales se puede representar mejor cuando se consideran en la ecuación constitutiva de los materiales derivadas de los esfuerzos y deformaciones de orden superior. Cuando se considera una excitación y respuesta armónica, de la forma $\tau=\tau_{0} \cdot e^{i \omega t}$ y $\gamma=\gamma_{0} \cdot e^{i \omega t}$ la ecuación constitutiva se puede expresar como:

$$
\gamma_{\mathrm{o}}=\left[G^{\prime}(\omega)+i \cdot G^{\prime \prime}(\omega)\right] \cdot \gamma_{\mathrm{o}}=\left[G^{*}(\omega) \cdot \gamma_{\mathrm{o}}\right.
$$

donde $G^{\prime}(\omega)$ y $G^{\prime \prime}(\omega)$ son los módulos de almacenamiento y de pérdida, respectivamente, y $G^{*}(\omega)$ se conoce como el módulo complejo. $G^{\prime}(\omega)$ es una medida de la energía almacenada y recuperada por ciclo y $G^{\prime \prime}(\omega)$ es una medida de la energía disipada por ciclo. Otra manera de expresar el módulo complejo es la siguiente:

$$
G^{*}(\omega)=G^{\prime}(\omega) \cdot\left(1+i \cdot \eta_{G}\right)
$$

donde $\eta_{G}$ es el factor de pérdida asociado con $G^{*}(\omega)$ :

$$
\eta_{G}=\frac{G^{\prime \prime}(\omega)}{G^{\prime}(\omega)}
$$


Este parámetro puede usarse para medir la capacidad de disipación de energía del material y se obtienen experimentalmente, de gráficas provistas por el fabricante del material o de gráficas disponibles en la literatura especializada, como Nashif et al. (1985) y Sun y Lu (1995). La Fig. 3 muestra la gráfica utilizada para obtener las propiedades viscoelásticas, el factor de pérdida y el módulo de almacenamiento, en función de la temperatura y la frecuencia para algunos de los modelos realizados en este trabajo.

FIG. 3 NOMOGRAMA DE UN POLÍMERO VISCOELÁSTICO 3M TM 110. (ADAPTADO DE LA GRÁFICA SUMINISTRADA POR EL FABRICANTE)

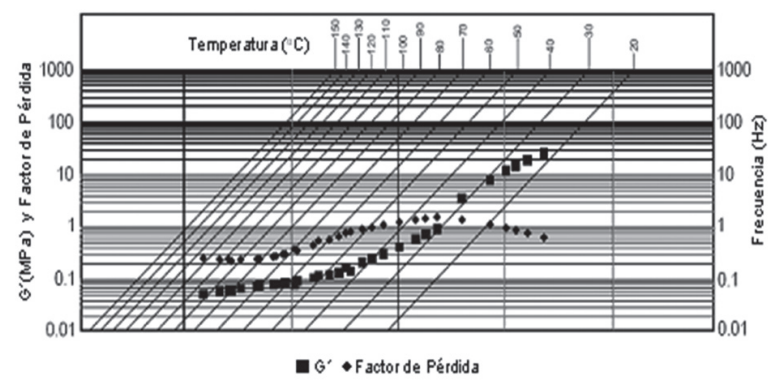

En esta investigación lo que se busca es establecer la efectividad de un tratamiento superficial de amortiguamiento, sin comparar respuestas sino mediante la determinación de los factores de pérdida globales que el tratamiento introduce en los modos más bajos. Por lo tanto, dado que se va a usar el programa ABAQUS para modelar estructuras con tratamiento de amortiguamiento, los factores de pérdida globales se obtienen con un procedimiento indirecto, el método de la media potencia (Craig Jr. 1981) el cual se describe a continuación.

\section{DETERMINACIÓN DE LAS FRECUENCIAS NATURALES $\omega_{\mathrm{N}}$ Y DEL FACTOR DE PÉRDIDA $\eta$.}

Un método muy usado para obtener las frecuencias naturales y el factor de pérdida de una viga, consiste en realizar un análisis de vibración forzada para una excitación armónica, en un rango de frecuencias que captura los modos de vibración de interés del sistema, con el fin de poder obtener la amplitud del desplazamiento vertical versus la frecuencia de la fuerza aplicada para un punto de la viga. La frecuencia natural amortiguada $\omega \mathrm{j}$ y el factor de pérdida modal del sistema nj para un determinado modo "j" se deducen aproximadamente de la curva de respuesta. La curva presenta una am- plitud máxima en cada frecuencia natural. Las frecuencias $\omega$ a y $\omega$ b por debajo y por arriba de una frecuencia natural en la cual la amplitud de la respuesta es $\sqrt{2 / 2}$ veces la amplitud de la frecuencia de resonancia se conocen como puntos de media potencia. Estas frecuencias se muestran en la Fig. 4 El factor de pérdida modal $\eta j$ se puede calcular aproximadamente usando el método de la media potencia, como la razón de la diferencia de las frecuencias $\omega \mathrm{b}$ y $\omega \mathrm{a}$ a la frecuencia natural amortiguada:

$$
\eta_{j}=\frac{\omega_{b}-\omega_{a}}{\omega_{j}}
$$

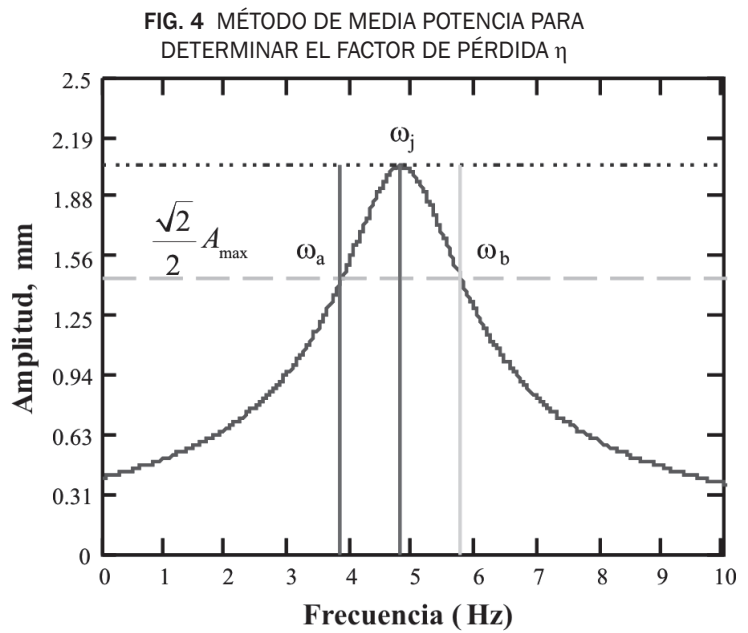

\section{FACTOR DE PÉRDIDA EN UNA VIGA CON TRATAMIENTO DE AMORTIGUAMIENTO A CORTANTE}

En 1978 Rao obtuvo unas expresiones numéricas para estimar las frecuencias naturales amortiguadas y los factores de pérdida modal en términos del llamado parámetro de cortante g, definido por la siguiente expresión:

$$
g=\frac{G_{2} A_{2} L^{2}}{t_{2}^{2}} \frac{\left(E_{1} A_{1}+E_{3} A_{3}\right)}{E_{1} A_{1}+E_{3} A_{3}}
$$

y por otro factor denominado factor geométrico $Y$ definido como:

$$
Y=\frac{c^{2}}{D} \frac{E_{1} A_{1} E_{3} A_{3}}{E_{1} A_{1}+E_{3} A_{3}}
$$

donde:

$A_{1}, A_{2}$ y $A_{3}$, son las áreas de la sección transversal de las capas elástica restringente, viscoelástica y elástica base, respectivamente. 
$E_{1}$ y $E_{3}$ son los módulos de elasticidad de las dos capas elásticas, restringente y base, respectivamente.

$\mathrm{L}$ es la longitud de la viga.

$\mathrm{D}=\mathrm{E}_{1} \mathrm{I}_{1}+\mathrm{E}_{3} \mathrm{I}_{3}$ es la rigidez flexural combinada de las dos capas elásticas.

c es la distancia entre los planos medios de las capas elásticas.

$G_{2}$ y $t_{2}$ son el módulo de rigidez a cortante y el espesor de la capa viscoelástica respectivamente.

A manera de ejemplo se presentan en la Tabla I las fórmulas encontradas por Rao para computar los factores de pérdida modal $\eta$ y de frecuencia $\Omega$ de una viga emparedado en voladizo vibrando en el primer modo. Las expresiones para otros modos de vibración y otras condiciones de frontera están disponibles en el artículo de Rao.

El factor de frecuencia $\Omega$ es un parámetro adimensional que permite calcular la frecuencia natural amortiguada como:

donde

$$
f=\frac{\Omega}{2 \pi t_{o}}
$$

$$
t_{o}=\sqrt{\frac{m L^{4}}{D}}
$$

siendo $m$ la masa por unidad de longitud de la viga emparedado.

TABLA I

FÓRMULAS DEL FACTOR DE FRECUENCIA Y DE PÉRDIDA PARA UNA VIGA EN VOLADIZO

\begin{tabular}{|c|c|}
\hline Modo & Fórmula \\
\hline \multirow{2}{*}{$\mathrm{I}$} & $\begin{array}{c}\eta=0.142675 \cdot 1.76507^{\mathrm{x}} \cdot 0.381063^{\mathrm{x}^{2}} \cdot 0.958039^{\mathrm{x}^{3}} \cdot 1.06549^{\mathrm{x}^{4}} \\
\end{array}$ \\
& $\begin{array}{c}\cdot 5.78986^{\mathrm{y}} \cdot 0.539055^{\mathrm{x} y} \cdot 1.08780^{\mathrm{x}^{2} \mathrm{y}} \cdot 1.08745^{\mathrm{x}^{3} \mathrm{y}} \cdot 0.976757^{\mathrm{x}^{4} \mathrm{y}} \\
\end{array}$ \\
& $\begin{array}{c}0.586965^{\mathrm{y}^{2}} \cdot 1.02241^{\mathrm{xy}} \cdot 1.1505^{\mathrm{x}^{2} \mathrm{y}^{2}} \cdot 0.97021^{\mathrm{x}^{3} \mathrm{y}^{2}} \cdot 0.983707^{\mathrm{x}^{4} \mathrm{y}^{2}} \cdot \eta_{2} \\
\end{array}$ \\
& $+\mathrm{y}\left(0.154016+0.553244 \mathrm{x}+0.144153 \mathrm{x}^{2}-0.075502 \mathrm{x}^{3}-0.014758 \mathrm{x}^{4}\right)$ \\
& $+\mathrm{y}^{2}\left(2.425840+2.142460 \mathrm{x}+0.294401 \mathrm{x}^{2}-0.194389 \mathrm{x}^{3}-0.048345 \mathrm{x}^{4}\right)$ \\
\end{tabular}

\section{MODELAMIENTO DE UNA COLUMNA CON TRATAMIENTO DE AMORTIGUAMIENTO. COLUMNA EN VOLADIZO.}

En esta sección se considera una columna W 14X109 con tratamiento viscoelástico a cortante en las dos superficies exteriores a sus alas y con una longitud $L=2,7432 \mathrm{~m}$ (9 pies). La columna se modela con el programa ABAQUS, con elementos finitos tridimensionales tipo C3D20: estos son elementos sólidos (tipo "ladrillo") de orden cuadrático con 20 nodos. En la Fig. 5 se muestra un esquema de la columna a modelar con dos capas viscoelásticas. La columna tiene su extremo inferior empotrado y su extremo superior libre. El modelo tiene en cuenta la variación de las propiedades mecánicas del material viscoelástico con respecto a la frecuencia de vibración. Estas pro- piedades se obtienen de la Fig. 3. El objetivo es estudiar el efecto del largo de la columna en el factor de pérdida $\eta$ y además comparar la variación de $\eta$ con el parámetro g de esta columna con la curva obtenida por Rao (1978) para una viga con las mismas condiciones de apoyo. Se consideran tres tratamientos a cortante los que generan tres secciones transversales identificadas como A, B y C. Los espesores $t_{1}$ para la capa restringente y $\mathrm{t}_{2}$ para la capa viscoelástica usados en cada sección se resumen en la Tabla II. Se calculó el factor de pérdida $\eta$ para diferentes valores de la longitud de la columna y los resultados encontrados para los tres primeros modos se muestran en las Fig. 6 a 9. Cada figura corresponde a uno de los tres primeros modos de vibración. En estas gráficas se puede observar que para cada sección transversal A, B y C y para cada modo de vibración existe 
una longitud específica para la cual se obtiene la mayor disipación de energía. Para poder identificar y apreciar mejor la longitud para la máxima disipación, las longitudes $L$ se variaron hasta 46 $\mathrm{m}$, si bien estos valores altos no tienen interés práctico. Identificar la longitud óptima para una sección dada es bien importante para conocer si se debe o no extender la longitud del tratamiento a toda la columna. También se puede observar en las Figuras 6 a 9 el máximo valor posible que puede alcanzar el factor de pérdida $\eta$. Esto es bien significativo pues nos permite predecir que para ciertas geometrías no será posible lograr una disipación de energía favorable con un determinado tratamiento a cortante.

FIG. 5 . COLUMNA CON TRATAMIENTO VISCOELÁSTICO

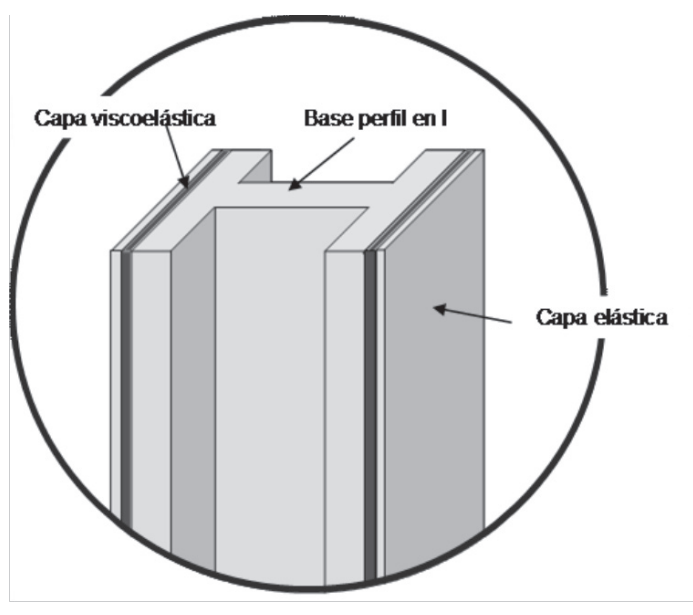

TABLA II

ESPESORES DEL TRATAMIENTO A CORTANTE PARA UNA COLUMNA W $14 X 109 \mathrm{FIJA}-$ LIB

\begin{tabular}{|c|c|c|}
\hline Sección & $\mathrm{t}_{1}(\mathrm{~mm})$ & $\mathrm{t}_{2}(\mathrm{~mm})$ \\
\hline $\mathrm{A}$ & 6.35 & 3.175 \\
\hline $\mathrm{B}$ & 6.35 & 22.225 \\
\hline C & 22.225 & 3.175 \\
\hline
\end{tabular}

FIG. 6 VARIACIÓN DEL FACTOR DE PÉRDIDA $\eta$ PARA EL PRIMER MODO DE UNA COLUMNA W 14 X 109 EMPOTRADA - LIBRE CON TRATAMIENTO A CORTANTE EN LAS DOS ALAS.

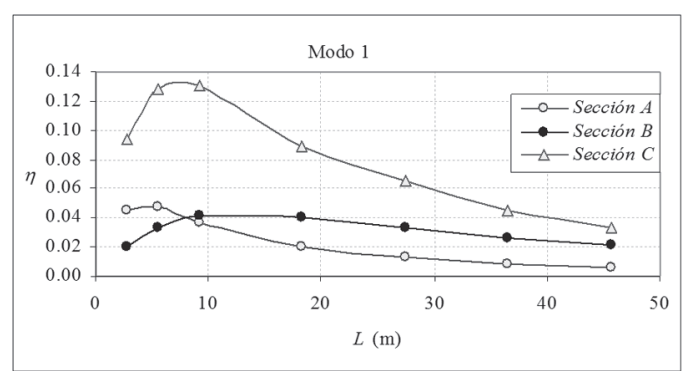

FIG. 7 VARIACIÓN DEL FACTOR DE PÉRDIDA $\eta$ PARA EL SEGUNDO MODO DE UNA COLUMNA W 14X109 EMPOTRADA - LIBRE CON TRATAMIENTO A CORTANTE EN LAS DOS ALAS

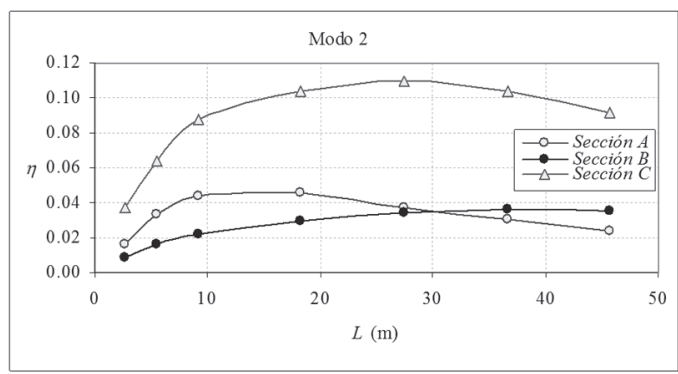

FIG. 8 VARIACIÓN DEL FACTOR DE PÉRDIDA $\eta$ PARA EL TERCER MODO DE UNA COLUMNA W 14X109 EMPOTRADA - LIBRE CON TRATAMIENTO A CORTANTE EN LAS DOS ALAS

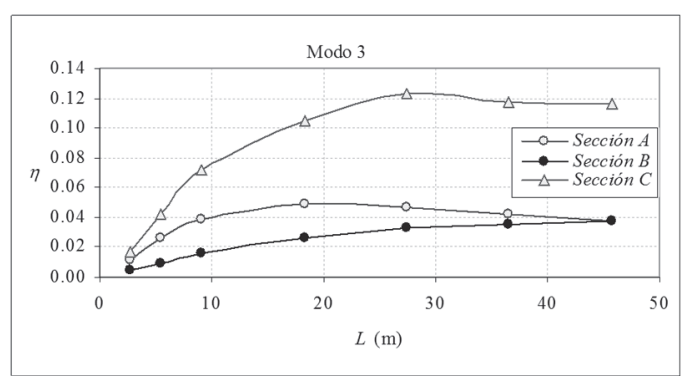

Ahora, si se calcula para las tres secciones transversales formadas con los tratamientos a cortante, el parámetro de cortante g y el factor geométrico $Y$ definidos en y respectivamente, se pueden obtener gráficas de $\eta$ versus g. Se obtienen así las curvas que se presentan en la Fig. 9 las que muestran el factor de pérdida $\mathrm{h}$ para el primer modo de vibración. Se nota que es favorable usar valores bajos para g, al igual que es deseable tener el valor más alto posible para Y.

Las expresiones obtenidas por Rao (1978) para el factor de pérdida $\eta$ como función de g y de Y que se presentaron en la Tabla I fueron derivadas para el caso donde se tiene un tratamiento a cortante en un solo lado de una viga en voladizo con una sección rectangular. Sin embargo, es interesante mostrar en una sola gráfica la curva obtenida con la fórmula numérica de Rao para el tratamiento a cortante en una cara junto con la curva calculada con el programa ABAQUS para tratamientos en ambas caras de la viga. Estas curvas se muestran en la Fig. 10 donde se observa que el valor máximo del factor de pérdida $\eta$ en ambos casos ocurre para un mismo valor de g aproximadamente. De este modo, la solución numérica de Rao podría uti- 
lizarse para estimar aproximadamente el espesor adecuado a utilizar en el tratamiento viscoelástico para una columna con el extremo libre.

FIG. 9 VARIACIÓN DEL FACTOR DE PÉRDIDA $\eta$ VS. EL PARÁMETRO DE CORTANTE G PARA UNA COLUMNA W 14X109 EMPOTRADO - LIBRE CON TRATAMIENTO A CORTANTE EN LAS DOS ALAS VIBRANDO EN EL PRIMER MODO

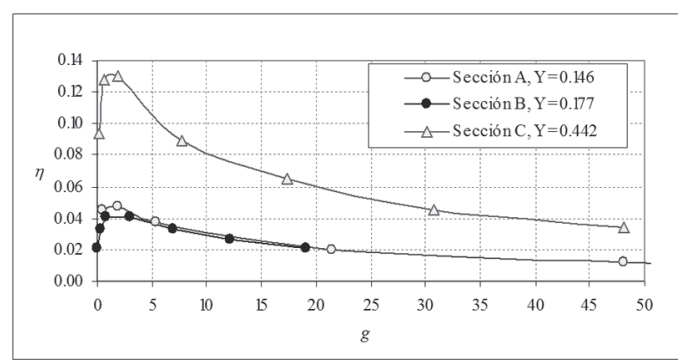

FIG. 10 COMPARACIÓN DEL FACTOR DE PÉRDIDA H PARA UNA VIGA W 14X109 CON TRATAMIENTO EN LAS DOS CARAS CON LA SOLUCIÓN NUMÉRICA PROPUESTA POR RAO PARA UNA VIGA DE SECCIÓN RECTANGULAR CON TRATAMIENTO EN UNA CARA VIBRANDO EN EL PRIMER MODO

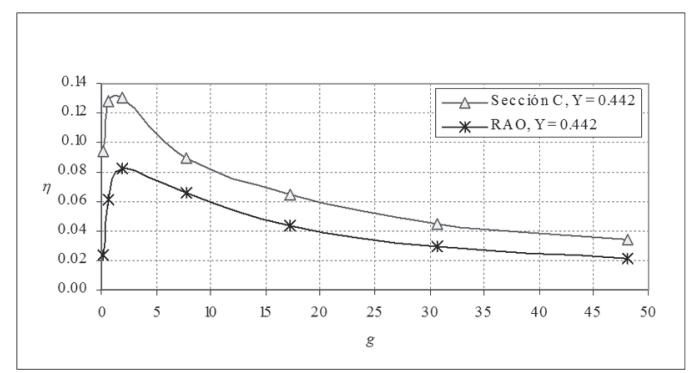

\section{GRÁFICA DEL FACTOR DE PÉRDIDA $\eta$ VS. EL PARÁMETRO DE CORTANTE G PARA UNA COLUMNA DE UN EDIFICIO DE CORTE}

Según las expresiones numéricas encontradas por Rao (1978) el factor de pérdida $\eta$ para una viga con tratamiento a cortante se puede calcular una vez que se dispone de los valores para el parámetro de cortante g y el factor geométrico $Y$. Las condiciones de frontera de la viga emparedado influyen en el valor del factor de pérdida $\eta$ que se puede lograr. Por lo tanto, es bien importante disponer de una gráfica del factor de pérdida $\eta$ para una condición de borde en particular debido a que a partir de ella es posible definir la región en la cual se logra la mayor disipación de energía. Estas curvas facilitan el proceso de diseño a la hora de seleccionar las dimensiones del tratamiento a cortante.

Para la condición de frontera que se muestra en la Fig. 11 no se dispone de la curva de variación del factor de pérdida $n$ con respecto al parámetro de cortante g. Como el objetivo de esta investigación es estudiar la factibilidad de aplicar los tratamientos de cortante en edificios de acero, es conveniente construir esta curva para poder usarla como guía a la hora de dimensionar el tratamiento a cortante. Por lo tanto, se modeló una columna con una sección W $14 \times 34$ y con una longitud de $2.7432 \mathrm{~m}$ en el programa ABAQUS, con elementos finitos en 3D tipo C3D20. Para construir la gráfica de $\eta$ vs. g se mantienen constantes las dimensiones $\left(A_{1}, A_{2}, A_{3}, t_{2}, L\right)$ de la viga emparedado y los módulos de Young $E_{1}$ y $E_{3}$ de los materiales elásticos, pero se permite variar al módulo de almacenamiento a cortante $G_{2}$ del material viscoelástico, de este modo, se pueden crear diferentes valores del parámetro de cortante g definido por. Debido a que no se cambia la geometría de la viga se obtiene un valor del factor geométrico $Y$ constante. El factor de pérdida del material viscoelástico $\eta 2$ se considera igual a 1. Para definir un punto de la gráfica $\eta$ vs. g, se escoge un valor para el parámetro de cortante g y se resuelve para $G_{2}$. En el modelo se consideró el módulo de almacenamiento a cortante $G_{2}$ independiente de la frecuencia. Para obtener los diferentes puntos de la curva $\eta$ vs. $g$, se fue calculando el valor de $G_{2}$ a partir de, con diferentes valores del parámetro a cortante g (0.1, $0.3,0.5,1,3,5,10,30,50,100)$. El tratamiento a cortante se aplicó en las dos caras externas del ala como se muestra en la Fig. 5. Para lograr diferentes valores del factor geométrico $Y$ se consideraron cinco combinaciones de espesores para el tratamiento a cortante, las que se muestran en la Tabla III, junto con los valores de Y obtenidos.

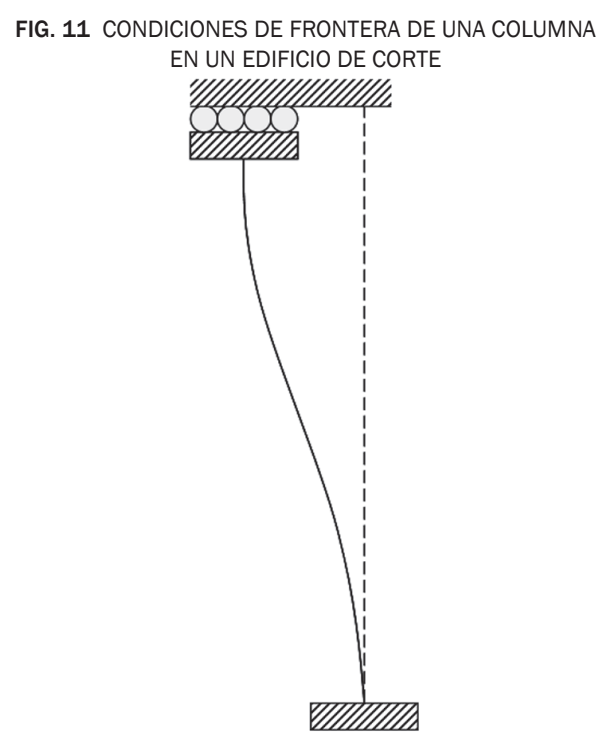


TABLA III

ESPESORES DEL TRATAMIENTO A CORTANTE PARA UNA COLUMNA W 14X34 DE UN EDIFICIO DE C

\begin{tabular}{|c|c|c|}
\hline $\mathrm{t}_{1}(\mathrm{~mm})$ & $\mathrm{t}_{2}(\mathrm{~mm})$ & $\mathrm{Y}$ \\
\hline 6.35 & 1.5875 & 0.222 \\
6.35 & 3.175 & 0.226 \\
6.35 & 12.7 & 0.250 \\
12.7 & 3.175 & 0.408 \\
12.7 & 12.7 & 0.451 \\
\hline
\end{tabular}

Los resultados encontrados para los tres primeros modos se presentan en las Fig. 12 a 15. Es importante notar que para esta longitud no es posible lograr para el factor geométrico $Y$ valores mayores que 1 , lo cual puede verse si se hace la gráfica del factor geométrico $Y$ versus el factor $a=A_{3} / A_{1}$. El valor máximo del factor de pérdida $\eta$ para el primer modo de vibración es aproximadamente 0.13 y está asociado a un valor de g aproximadamente igual a 7 como se muestra en la Fig. 12. En las Fig. 13 y 15 se puede observar que los valores máximos para el factor de pérdida $\eta$ para el segundo y tercer modo se obtienen para valores aproximadamente de $\mathrm{g}=30$ y $\mathrm{g}=40$ respectivamente. Estos valores máximos de $\eta$ para el segundo y tercer modo son 0.08 y 0.07 respectivamente.

FIG. 12 VARIACIÓN DEL FACTOR DE PÉRDIDA $\eta$ DEL PRIMER MODO PARA UNA COLUMNA W 14X34 DE UN EDIFICIO DE CORTE CON TRATAMIENTO EN LAS DOS CARAS OBTENIDA CON ABAQUS

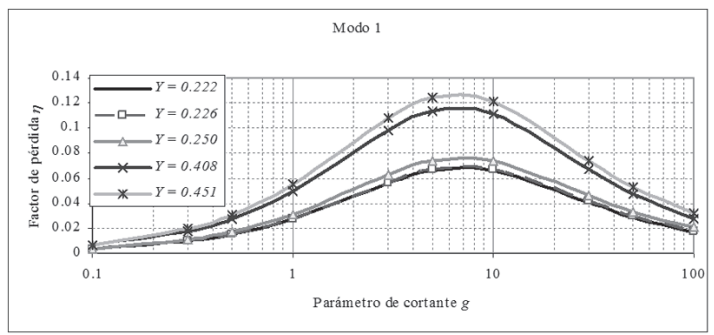

FIG. 13 VARIACIÓN DEL FACTOR DE PÉRDIDA $\eta$ DEL SEGUNDO MODO PARA UNA COLUMNA W 14X34 DE UN EDIFICIO DE CORTE CON TRATAMIENTO EN LAS DOS CARAS OBTENIDA CON ABAQUS

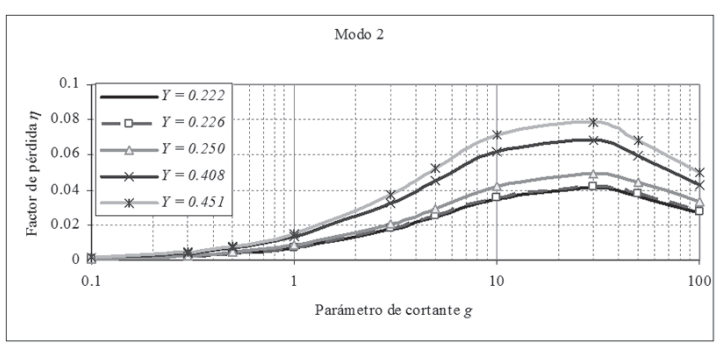

FIG. 14 VARIACIÓN DEL FACTOR DE PÉRDIDA $\eta$ DEL TERCER MODO PARA UNA COLUMNA W 14X34 DE UN EDIFICIO DE CORTE CON TRATAMIENTO EN LAS DOS CARAS OBTENIDA CON ABAQUS

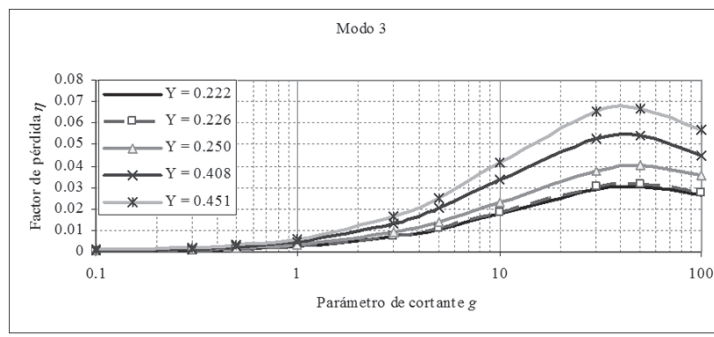

Se debe mencionar que es posible obtener curvas para valores de $Y$ mayores a los presentados en las Fig. 12 a 14 con una columna de mayor longitud. Sin embargo, puesto que se desea usar estas gráficas para columnas de edificios, las cuales tienen una altura de alrededor de $2.8 \mathrm{~m}$, las curvas para valores mayores de $Y$ no tendrán interés práctico.

\section{MODELAMIENTO DE UNA ESTRUCTURA APORTICADA CON TRATAMIENTO A CORTANTE}

Para estudiar la efectividad del tratamiento a cortante en la disipación de energía en una estructura aporticada, en esta sección se modelaron con elementos finitos tres estructuras de edificios de uno, dos y tres pisos, formados con perfiles de acero en forma de I. Cada estructura se modeló como un edificio de corte. También se muestran los resultados encontrados al modelar un pórtico de nueve pisos sin restringir la estructura a comportarse como un edificio de corte

A. Procedimiento para dimensionar el tratamiento a cortante.

El tratamiento a cortante se aplica a las columnas en las caras externas de cada ala, como se muestra en la Fig. 5. Para determinar los espesores utilizados tanto en la capa restringente como en la capa viscoelástica de cada uno de los modelos creados en el programa ABAQUS se siguió el siguiente procedimiento:

\section{Paso 1. Selección del espesor de la capa res- tringente $t_{1}$}

Para una columna de un pórtico típico para obtener valores altos del factor geométrico $Y$ se requiere tomar la relación $A_{3} / A_{1}=1$, lo cual a su vez implica utilizar para la capa restringente un espesor muy grande (Gil y Suárez 2007). Sin embargo, 
puesto que no es práctico usar valores exagerados ni en la capa viscoelástica ni en la capa restringente, se hace necesario definir unos espesores racionales para el espesor $t_{1}$ y evaluar su efectividad en el amortiguamiento estructural que se logra.

\section{Paso 2. Cálculo de la frecuencia natural del pórtico}

Las propiedades de los materiales viscoelásticos cambian con la frecuencia. Sin embargo, puesto que en los diseños sísmicos de estructuras regulares se tiene en cuenta el primer modo de vibración, es razonable utilizar para propósitos de dimensionar el tratamiento a cortante la frecuencia natural del pórtico asociada a este modo. Por lo tanto, es necesario calcular la frecuencia natural del primer modo de vibración de cada modelo.

\section{Paso 3. Determinación del módulo de almace- namiento $\mathbf{G}_{\mathbf{2}}$}

Con el valor de la frecuencia natural fundamental encontrada en el paso anterior y usando una gráfica de las propiedades del material viscoelástico provista por el fabricante se puede determinar el módulo de almacenamiento a cortante $G_{2}$. En esta investigación se obtuvo de la Fig. 3.

\section{Paso 4. Establecimiento del espesor de la capa viscoelástica $\mathbf{t}_{2}$}

El espesor de la capa viscoelástica se puede determinar a partir de y con las curvas encontradas en la parte $\mathrm{VI}$, para el factor de pérdida $\eta$ con respecto al parámetro de cortante g, para poder así seleccionar el valor del parámetro de cortante g asociado a la mayor disipación de energía. La curva correspondiente a un $\mathrm{h}$ para el primer modo se presentó en la Fig. 12. Esta gráfica se muestra que el valor de g para lograr la mayor disipación es aproximadamente igual a 7 . Por lo tanto, para determinar el valor del espesor de la capa viscoelástica $t_{2}$ de cada modelo usando se puede tomar allí un valor de $g=7$.

Para encontrar el factor de pérdida $\eta$ se aplica a cada modelo construido en el programa ABAQUS el método de la media potencia que se explicó en la parte III.

\section{B. Pórticos modelados como edificios de corte.}

En el programa ABAQUS se modeló tres pórticos de 1, 2 y 3 pisos con tratamiento a cortante en las columnas. El área en planta de los pórticos estudiados es de $6 \mathrm{~m}$ por $6 \mathrm{~m}$. Para las columnas se consideró una altura típica de 2.7432 m (9 pies). Las cargas muertas y vivas que actúan en los pisos son $5 \mathrm{kPa}$ y $2 \mathrm{kPa}$, respectivamente. Además de la masa propia del pórtico se consideró la masa asociada a las cargas muertas y aquella correspondiente a un $25 \%$ de las cargas vivas que actúan sobre la viga. Esta masa se distribuyó de manera uniforme en el volumen de la viga. Para simplificar la elaboración del modelo se consideraron dos perfiles en cada pórtico. Se utilizó un perfil W 18X35 en las vigas y una sección W 14X34 para las columnas. También se supone que los pórticos se comportan como un edificio de corte. Este comportamiento se simuló en el programa ABAQUS asignando un valor alto al módulo de elasticidad $\mathrm{E}$ de las vigas.

Aplicando el procedimiento descrito en la sección anterior a los pórticos de 1, 2 y 3 pisos, se especificó el tratamiento a cortante a ser utilizado en los modelos construidos en el programa ABAQUS. En la Tabla IV se resumen los resultados.

TABLA IV

RESULTADOS DEL DIMENSIONAMIENTO DEL TRATAMIENTO A CORTANTE PARA LOS PÓRTICOS DE 1, 2 Y 3 PISOS

\begin{tabular}{|c|c|c|c|c|}
\hline Pórtico & paso 1 & paso 2 & paso 3 & paso 4 \\
\hline No. Pisos & $\mathrm{t}_{1}(\mathrm{~mm})$ & $\omega_{\mathrm{n}}(\mathrm{Hz})$ & $\mathrm{G}_{2}(\mathrm{kPa})$ & $\mathrm{t}_{2}(\mathrm{~mm})$ \\
\hline 1 & 12.7 & 7.3 & 265.33 & 0.15 \\
\hline 2 & 12.7 & 4.0 & 211.00 & 0.12 \\
\hline 3 & 12.7 & 2.7 & 176.60 & 0.10 \\
\hline
\end{tabular}

En la Tabla $V$ se presentan los resultados encontrados para el factor de pérdida $\eta$ de cada modelo construido en el programa ABAQUS. La tabla muestra las frecuencias $\omega_{a}$ y $\omega_{b}$ asociadas a los puntos de media potencia que se usaron para calcular los factores de pérdida modales. El mayor valor del factor de pérdida $\mathrm{h}$ en el primer modo de vibración se presenta en el pórtico de un piso. Sin embargo, el valor más alto del factor de pérdida se logra para el tercer modo de vibración en el pórtico de tres pisos. La razón de se debe a que en el tercer modo de vibración, el material viscoelástico de las columnas está sometido a una mayor deformación por cortante.

TABLA V

PROPIEDADES DINÁMICAS DE LOS PÓRTICOS DE 1, 2 Y 3 PISOS EN ESTUDIO

\begin{tabular}{|c|c|c|c|c|c|}
\hline $\begin{array}{c}\text { Pórtico } \\
\text { No. Pisos }\end{array}$ & Modo & $\omega_{a}(\mathrm{~Hz})$ & $\omega_{b}(\mathrm{~Hz})$ & $\omega_{j}(\mathrm{~Hz})$ & $\eta_{j}$ \\
\hline 1 & 1 & 7.94 & 8.60 & 8.28 & 0.079 \\
\hline 2 & 1 & 4.38 & 4.64 & 4.51 & 0.057 \\
\hline & 2 & 14.01 & 15.30 & 14.51 & 0.089 \\
\hline 3 & 1 & 2.92 & 3.06 & 2.99 & 0.047 \\
\hline & 2 & 9.50 & 10.12 & 9.77 & 0.063 \\
\hline & 3 & 16.53 & 18.72 & 17.21 & 0.127 \\
\hline
\end{tabular}




\section{Pórtico de 9 pisos con tratamiento a cortante.}

A continuación se presentan los resultados encontrados al modelar en el programa ABAQUS un pórtico de 9 pisos con un tratamiento a cortante en las columnas. Se usaron la misma área en planta, altura de piso y las cargas del modelo anterior de tres pisos. También se hizo la misma consideración para computar y distribuir la masa de la estructura en ABAQUS. No se restringió la estructura a comportarse como un edificio de corte. Se usó un perfil W 18X35 para todas las vigas y una sección W 14X34 para todas las columnas. Con la intención de identificar el espesor de la capa viscoelástica $t_{2}$ que maximiza la disipación de energía, se modelaron ocho pórticos con diferentes espesores $t_{2}$. Estos espesores se presentan en la Tabla VI junto con las frecuencias naturales de los primeros tres modos. El espesor de la capa restringente $t_{1}$ se consideró como la mitad del espesor del ala de la columna $\quad\left(\mathrm{t}_{1}=5.7785\right.$ $\mathrm{mm}$ ). Para graficar el factor de pérdida $\mathrm{h}$ con respecto al parámetro de cortante g, se procedió de manera similar al pórtico de tres pisos, es decir, se calculó la frecuencia promedio de cada modo y se tomó un valor aproximado a cada una de ellas para después determinar con los datos de la Fig. 3 el módulo de almacenamiento a cortante $G_{2}$. La Tabla VI resume los valores de frecuencia utilizados y los respectivos valores de $G_{2}$.

Los valores obtenidos para el factor geométrico Y para distintos espesores de la capa viscoelástica $t_{2}$ se resumen en la Tabla VII. En esta tabla también se muestran los valores del parámetro de cortante g y el factor de pérdida $\mathrm{h}$ alcanzado con cada tratamiento en el primer modo. De manera similar al pórtico de tres pisos, se nota que aún cuando se usó un espesor de capa viscoelástica muy alto, $\mathrm{t}_{2}=190.95 \mathrm{~mm}$, no se logró un valor favorable para el factor de pérdida $(\eta=0.021)$. En la Tabla VII también se puede notar que el espesor de la capa viscoelástica $\left(\mathrm{t}_{2}=0.090 \mathrm{~mm}\right)$ asociado a la mayor disipación de energía (cuando $g=$ 8.365) es muy bajo relativo al espesor de la placa restringente $\left(\mathrm{t}_{1}=5.7785 \mathrm{~mm}\right)$ y al espesor del ala de la columna $\left(t_{f}=11.557 \mathrm{~mm}\right)$. Además en esta tabla se puede observar que el parámetro de cortante g es más sensible que el factor geométrico $Y$ a los cambios en el espesor de la capa viscoelástica $t_{2}$. En la Fig. 15 se muestra cómo varía el factor de pérdida $\mathrm{h}$ con respecto al parámetro de cortan- te $g$ para los tres primeros modos. De nuevo en esta figura se puede notar que el mayor valor para el factor de pérdida se presenta en el tercer modo. Sin embargo, su valor es más bajo comparado con el logrado para el pórtico de tres pisos.

TABLA VI

ESPESOR DE LA CAPA VISCOELÁSTICA $t_{2}$, FRECUENCIAS NATURALES Y MÓDULO DE RIGIDEZ A CORTANTE G, EN UN PÓRTICO DE 9 PISOS CON TRATAMIENTO A CORTANTE

\begin{tabular}{|c|c|c|c|}
\hline $\mathrm{t}_{2}(\mathrm{~mm})$ & $\omega_{1}(\mathrm{~Hz})$ & $\omega_{2}(\mathrm{~Hz})$ & $\omega_{3}(\mathrm{~Hz})$ \\
\hline 190.950 & 0.606 & 1.943 & 3.585 \\
19.095 & 0.622 & 1.959 & 3.552 \\
3.800 & 0.625 & 1.968 & 3.564 \\
1.900 & 0.626 & 1.973 & 3.573 \\
0.950 & 0.628 & 1.979 & 3.588 \\
0.380 & 0.631 & 1.993 & 3.627 \\
0.175 & 0.635 & 2.014 & 3.676 \\
0.090 & 0.640 & 2.033 & 3.716 \\
\hline Frecuencia Promedio & 0.627 & 1.983 & 3.610 \\
\hline Frecuencia Utilizada & 0.6 & 2.0 & 3.6 \\
\hline G2 (kPa) & 100 & 161 & 201.4 \\
\hline
\end{tabular}

TABLA VII

ESPESOR DE LA CAPA VISCOELÁSTICA $t_{2}$, FACTOR GEOMÉTRICO $Y$, PARÁMETRO DE CORTANTE Y FACTOR DE PÉRDIDA $\eta_{3}$ PARA EL PRIMER MODO EN UN PÓRTICO DE 9 PISOS CON TRATAMIENTO A CORTANTE

\begin{tabular}{|c|c|c|c|}
\hline $\mathrm{t}_{2}(\mathrm{~mm})$ & $Y$ & $g$ & $\eta_{1}$ \\
\hline 190.950 & 0.850 & 0.004 & 0.021 \\
19.095 & 0.245 & 0.039 & 0.021 \\
3.800 & 0.209 & 0.198 & 0.022 \\
1.900 & 0.205 & 0.396 & 0.022 \\
0.950 & 0.203 & 0.792 & 0.023 \\
0.380 & 0.202 & 1.981 & 0.026 \\
0.175 & 0.201 & 4.302 & 0.029 \\
0.090 & 0.201 & 8.365 & 0.031 \\
\hline
\end{tabular}

FIG. 15 VARIACIÓN DEL FACTOR DE PÉRDIDA $\eta$ EN UN PÓRTICO DE NUEVE PISOS

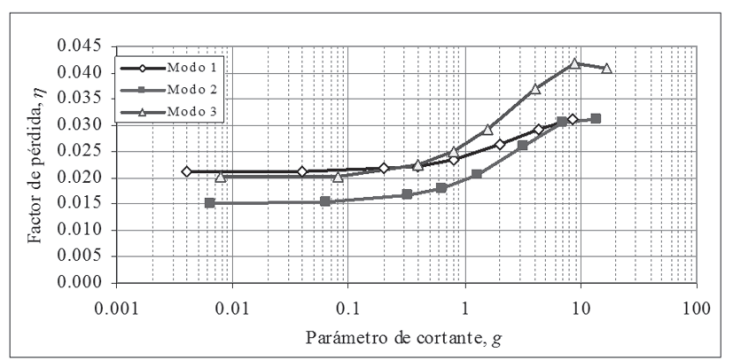




\section{CONCLUSIONES}

Con base en los resultados del estudio realizado en esta investigación en las columnas y pórticos con tratamiento a cortante se obtienen las siguientes conclusiones:

Se mostró que los valores mayores del espesor de la capa viscoelástica en un tratamiento a cortante no necesariamente implican un aumento en la disipación de energía. En un tratamiento a cortante el parámetro de cortante g es más sensitivo que el factor geométrico $\mathrm{Y}$ a los cambios en el espesor de la capa viscoelástica $t_{2}$. Para las longitudes típicas que se usan en los pórticos de acero construidos con perfiles en forma de I, la configuración para el tratamiento a cortante asociada a la mayor disipación de energía que se obtuvo tenía espesores tanto para el material viscoelástico como para la placa restringente irreales. El estudio de pórticos modelados mostró valores bajos para los factores de pérdida, lo cual permitió concluir que el uso de tratamientos de amortiguamiento en las columnas de edificios de acero no se considera como alternativa viable para reducir la respuesta a cargas dinámicas.

\section{REFERENCIAS}

[1] J. F. Fuller, E. F. Fuchs, and K. J. Roesler, "Influence of harmonics on power distribution system protection," IEEE Trans. Power Delivery, vol. 3, no.2, pp. 549-557, Apr. 1988

[2] Alberts, T. E., Xia, H., and Chen, Y. (1992). "Dynamic Analysis to Evaluate Viscoelastic Passive Damping Augmentation for the Space Shuttle Remote Manipulator System." Transactions of the ASME, Dynamics Systems, Measurement, and Control, Vol. 114(3), 468-475

[3] DiTaranto, R. A. (1965). "Theory of Vibratory Bending for Elastic and Viscoelastic Layered Finite-Length Beams." Journal of Applied Mechanics, 32, 881-886

[4] Flugge, W. (1967). Viscoelasticity, Blaisdell Publishing Company, Waltham, Massachusetts

[5] Gil, J. J., and Suárez, L. E. "Efecto de la Geometría de Vigas con Capas Viscoelásticas en la Disipación de Energía." ENIEF 2007 XVI Congreso sobre Métodos Numéricos y sus Aplicaciones, Ciudad de Córdoba, Córdoba, Argentina

[6] Johnson, C. D., and Kienholz, D. A. (1982). "Finite Element Prediction of Damping in Structures with Constrained Viscoelastic Layers." AIAA Journal, 20(9), 1284-1290
[7] Lifshitz, J. M., and Leibowitz, M. (1987). "Optimal Sandwich Beam Design for Maximum Viscoelastic Damping." International Journal of Solids and Structures, Vol. 23(7), 1027-1034

[8] Luengo, P. "Design of Joints for Damped Structures." Proceedings of the International Conference: Spacecraft Structures and Mechanical Testing, Noordwijk, The Netherlands, 24-26

[9] Lumsdaine, A., and Scott, R. A. (1995). "Shape Optimization of Unconstrained Beam and Plate Damping Layers." Design Engineering Technical conferences, Vol. 3 - Part C, 15 - 22

[10] Lunden, R. (1980). "Optimun Distribuction of Additive Damping for Vibrating Frames." Journal of Sound and Vibration, Vol 72(3), 391-402

[11] Macé, M. (1994). "Damping of Beam Vibrations by Means of a Thin Constrained Viscoelastic Layer: Evaluation of a New Theory." Journal of Sound and Vibration, 172(5), 577-591

[12] Marceling, J.-L., Trompetti, P., and A. Smati, A. (1992). "Optimal Constrained Layer Damping with Partial Coverage." Finite Elements in Analysis and Design, Vol. $12,273-280$

[13] Mead, D. J., and Markus, S. (1969). "The Forced vibration of a Three-layer, Damped Sandwich Beam with Arbitrary Boundary Conditions." Journal of Sound and Vibration, 10(2), 163-175

[14] Oberts, V. H. (1952). “Uber die Damfung der Biegeschwingungen Dunnner Blechedurch Fest Haftende Belage." Acustica Vol. 2, 181-194

[15] Plunkett, R., and Lee, C. T. (1969). "Length Optimization for Constrained Viscoelastic Layer Damping." The Journal of the Acoustical of America, Vol. 48(1), 150161

[16] Rao, D. K. (1978). "Frequency and Loss Factors of Sandwich Beams Under Various Boundary Conditions." Journal of Mechanical Engineering Science, 20(5), 271-282

[17] Ross, D., Ungar, E. E., and Kerwin, E. M. (1959). "Damping of Plate Flexural Vibrations by Means of Viscoleastic Laminae." Structural Damping, Section 3,ASME Monograph on Structural Damping, New York, $49-88$

[18] Roy, P. K., and Ganesan. (1996). "Dynamic Studies on Beams with Unconstrained layer Damping Treatment." Journal of Sound and Vibration, Vol. 195(3), 417-427

[19] Soni, M. L., and Bogner, F. K. (1982). "Finite Element Vibration Analysis of Damped Structures." AIAA Journal, 20, 700-707 
[20] Sun, C. T., Sankar, B. V., and Rao, V. S. (1990). "Damping and vibration control of unidirectional composite laminates using add-on viscoelastic materials." Journal of Sound and Vibration, Vol. 139(2), 277-287

[21] Yildiz, A., and Stevens, K. (1985). "Optimum Thickness Distribution of Unconstrained Viscoelastic Damping Layer Treatments for Plates." Journal of Sound and Vibration, Vol. 103(2), 183-199

[22] E. Clarke, Circuit Analysis of AC Power Systems, vol. I. New York: Wiley, 1950, p. 81

[23] Brighem, E. O. (1988). The Fast Fourier Transform and its Applications, Prentice Hall, Englewood Cliff, New Jersey

[24] Craig Jr., R. R. (1981). Structural Dynamics an Introduction to Computer methods, john Wiley \& Sons, New York

[25] Christensen, R. M. (1971). Theory of Viscoelasticity: an Introduction, Academic Press, New York

[26] Nashif, A. D., Jones, D. I. G., and Henderson, J. P. (1985). Vibration Damping, John Wiley \& Sons, New York

[27] Sun, C. T., and Lu, Y. P. (1995). Vibration Damping of Structural Elements, Prentice Hall PRT, Englewood Cliffs, New Jersey 